\title{
EFEITO PROTETOR DE ANTAGONISTA DAS GLICOPROTEÍNAS IIb/IIIa NAS ALTERAÇÕES HEPÁTICAS E PULMONARES SECUNDÁRIAS À ISQUEMIA E REPERFUSÃO DE FÍGADO EM RATOS
}

\author{
Leonardo F. CANEDO, Marcel Autran C. MACHADO, Ana M. M. COELHO, \\ Sandra N. SAMPIETRE, Telesforo BACCHELLA e Marcel C. C. MACHADO
}

\begin{abstract}
RESUMO - Racional - A lesão de isquemia e reperfusão hepática é um evento comum e responsável por considerável morbidade e mortalidade. Objetivo - Avaliar efeitos de inibidor da glicoproteína IIb/IIIa, cloridrato de tirofiban, nas alterações hepáticas e pulmonares da lesão de isquemia e reperfusão de fígado de ratos. Método - Vinte e três ratos Wistar divididos em três grupos: laparotomia $(\mathrm{n}=6)$, isquemia e reperfusão que receberam solução fisiológica $(n=8)$, e submetidos a isquemia e reperfusão e tratados com o cloridrato de tirofiban $(n=9)$. Foram realizadas dosagens das aminotransferases e análise histológica hepática. Avaliação pulmonar foi realizada pelo teste do azul de Evans e pela dosagem tecidual da mieloperoxidase no parênquima pulmonar. A oxidação e fosforilação mitocondrial das células hepáticas também foram avaliadas. Resultados - O grupo tratado com cloridrato de tirofiban apresentou menores níveis de aminotransferases, assim como alterações histológicas menos intensas. Avaliação pulmonar demonstrou diminuição no teste de azul de Evans no grupo tratado com cloridrato de tirofiban. Grupo tratado com cloridrato de tirofiban apresentou aumento significativo do estado 3 da respiração mitocondrial e das relações adenosina difosfato utilizado para fosforilação sobre o oxigênio consumido na reação e de coeficiente respiratório. Conclusões - O uso do cloridrato de tirofiban exerceu papel protetor da lesão hepática de isquemia e reperfusão e impediu o aumento da permeabilidade vascular secundária à lesão de reperfusão hepática.
\end{abstract}

DESCRITORES - Traumatismo por reperfusão. Fígado. Hepatectomia. Glicoproteínas da membrana de plaquetas. Ratos.

\section{INTRODUÇÃO}

A lesão de isquemia e reperfusão é a principal determinante da disfunção hepática após hepatectomias, e também, a causa mais importante da disfunção primária do enxerto hepático ${ }^{(2,}$ ${ }^{3,23)}$, além de ser a principal responsável pelo retransplante nas primeiras duas semanas de pós-operatório ${ }^{(9)}$.

Sabe-se que as plaquetas contribuem para lesão de isquemia e reperfusão no coração $0^{(8)}$, pulmão $^{(20)}$ e pâncreas ${ }^{(15)}$. No fígado, o bloqueio da agregação plaquetária também demonstrou efeito protetor na microcirculação ${ }^{(14)}$.

As plaquetas carreiam várias moléculas de adesão necessárias às interações intercelulares como a P-selectina, as integrinas e as glicoproteínas $\mathrm{Ib}$ e $\mathrm{IIb} / \mathrm{IIIa}^{(16)}$. As glicoproteínas Ib são essenciais para a adesão plaquetária à matriz subendotelial, formando ligações com o fator de Von Willebrand. As glicoproteínas IIb/IIIa, através de ligações com o fibrinogênio, formam as adesões entre as plaquetas, promovendo a agregação plaquetária( ${ }^{(13)}$.

Apesar de a agregação plaquetária ser mediada pelas diversas moléculas de adesão, a etapa final é a ligação do fibrinogênio à plaqueta ativada, sendo o complexo glicoprotéico de membrana IIb/IIIa o receptor do fibrinogênio ${ }^{(21,22)}$. Assim, as glicoproteínas IIb/IIIa representam um alvo importante na supressão da função plaquetária de agregação, com o benefício de minimizar os riscos de sangramento, uma vez que o seu bloqueio não impede a adesão das plaquetas ao endotélio lesionado.

O cloridrato de tirofiban é um antagonista de baixo peso molecular específico para os receptores das glicoproteínas $\mathrm{IIb} / \mathrm{III}$. O presente estudo teve o objetivo de avaliar a ação do cloridrato de tirofiban na lesão de isquemia e reperfusão do fígado, analisando as alterações hepáticas e pulmonares.

\section{MÉTODOS}

Foram utilizados ratos Wistar, com peso entre 200 e $250 \mathrm{~g}$, criados no Biotério Central da Faculdade de Medicina da Universidade de São Paulo, São Paulo, SP. Todos os animais foram tratados segundo as Normas Internacionais de Proteção aos Animais, sendo anestesiados com solução de cloridrato de cetamina e cloridrato de xylasina na razão 4:1 (Ketalar ${ }^{\circledR} 50 \mathrm{mg} . \mathrm{cm}^{3} /$ Rompum $^{\circledR}$ ) administrada pela via intraperitonial. Os ratos foram posicionados na mesa cirúrgica em decúbito dorsal e imobilizados pelas quatro extremidades e pelo maxilar superior. Foi realizada tricotomia

Trabalho realizado no LIM-37 do Departamento de Gastroenterologia da Faculdade de Medicina da Universidade de São Paulo, SP.

Correspondência: Dr. Marcel Autran C. Machado - Rua Evangelista Rodrigues, 407 - 05463-000 - São Paulo - Brasil. E-mail: dr@drmarcel.com.br 
da região abdominal, seguida de aplicação de solução anti-séptica. A glote foi visualizada pela tração da língua, para fora e para cima, e transluminescência da região (Figura 2). Um cateter $\left(\right.$ Jelco $\left.^{\circledR}\right)$ número 14 adaptado foi utilizado como cânula orotraqueal. $\mathrm{O}$ ventilador mecânico (Harvard inc., Holliston, MA, EUA) foi acoplado e ajustado para 60 incursões ventilatórias por minuto com volume corrente de $0,08 \mathrm{~mL}$ por grama de peso. O termômetro retal (YSI inc., Dayton, Ohio, EUA) foi posicionado e fixado.

Feita incisão mediana da pele e secção dos ligamentos hepáticos, a seguir exposição do hilo, isolamento do pedículo comum aos lobos mediano e ântero-lateral, e colocação de pinça microvascular atraumática. Constatada a mudança de coloração dos lobos hepáticos isquêmicos, delimitando uma linha de transição com a região do fígado não-isquêmica, procedeu-se, finalmente, ao fechamento da parede abdominal em plano único para minimizar as perdas de calor e água. Os animais foram submetidos a 1 hora de isquemia quente a partir do pinçamento do pedículo ${ }^{(24)}$. Durante esse período, os animais foram mantidos anestesiados, em ventilação mecânica e sob controle da temperatura, mantida entre $36,5^{\circ} \mathrm{C}$ e $37^{\circ} \mathrm{C}$. Após 45 minutos de isquemia quente, foi injetado pela veia peniana dorsal $1 \mathrm{~mL}$ de solução salina a $0,9 \%$ ou droga teste. Após 1 hora de isquemia quente, o rato foi reoperado e a pinça vascular retirada cuidadosamente, finalizando-se o período de isquemia e iniciando-se o período de reperfusão, que foi certificada pela mudança da coloração.

Os ratos foram extubados após a retomada da ventilação espontânea e reflexo ao estímulo doloroso em membros inferiores e posteriormente acondicionados em gaiolas individuais com água ad libitum. Após 4 horas de reperfusão, os animais foram novamente anestesiados utilizando-se $2 \mathrm{~mL}$ da solução anestésica (cetamina/xylasina). Os ratos foram posicionados novamente na mesa cirúrgica, e foi injetado 0,5 mL de azul de Evans pela veia peniana dorsal. Passados 15 minutos, realizou-se toracolaparotomia, punção cardíaca para coleta de $1 \mathrm{~mL}$ de sangue e secção da veia cava inferior.

O fígado foi retirado para estudo histológico e da respiração mitocondrial. $\mathrm{O}$ coração foi seccionado no ventrículo direito e passado um cateter de silicone de 2,0 mm de diâmetro (Silastic, Dow Corning, $\mathrm{n}^{\circ}$ 602.175) pelo tronco da artéria pulmonar, e injetados $100 \mathrm{~mL}$ de solução salina $0,9 \%$ a $10 \mathrm{~mL} /$ minuto para retirada do sangue intravascular. Os pulmões foram ressecados e encaminhados para estudo da atividade tecidual da mieloperoxidase e da densidade óptica do Azul de Evans.

Os animais foram divididos em 3 grupos: grupo 1 (G1) - seis ratos foram submetidos a laparotomia sem pinçamento do pedículo. Grupo 2 (G2) - oito ratos foram submetidos a isquemia e reperfusão, e receberam a administração endovenosa de $1 \mathrm{~mL}$ de solução fisiológica 0,9\%. Grupo 3 (G3) - nove ratos foram submetidos a isquemia e reperfusão e receberam a administração endovenosa de $1 \mathrm{~mL}$ de cloridrato de tirofiban $(0,7 \mathrm{mg} / \mathrm{kg})$. O cloridrato de tirofiban (Agrastat ${ }^{\circledR}$ ), apresentado em frasco-ampola com $12,5 \mathrm{mg}$ e $50 \mathrm{~mL}$, foi diluído em soro fisiológico a $0,9 \%$.

\section{ESTUDOS REALIZADOS}

\section{Bioquímica (AST e ALT)}

As aminotransferases foram utilizadas como marcador de lesão hepática.

\section{Respiração mitocondrial}

A função mitocondrial do fígado foi estudada segundo metodologia descrita anteriormente ${ }^{(4)}$. O consumo de oxigênio pela mitocôndria foi determinado polarograficamente ${ }^{(7)}$, usando um oxígrafo 5/6 (Gilson Medical Eletronics, Inc.) com eletrodo de $\mathrm{O}_{2}$ (Clark, Yellow Springs Instruments Co.; Yellow Springs, Ohio, EUA) a $28^{\circ} \mathrm{C}$. Para a determinação do estado 4 da respiração (estado basal) as mitocôndrias receberam como substrato energético o succinato de potássio. O estado 3 (S3) da respiração (estado ativo) foi induzido pela adição de adenosina difosfato (ADP, Sigma Chemical Company, St. Louis, Missouri, EUA). Após fosforilação completa de todo ADP adicionado para ATP, o estado 4 (S4) da respiração foi medido novamente.

A relação de controle respiratório avalia o acoplamento das reações mitocondriais pela razão de $\mathrm{S} 3 \mathrm{com} \mathrm{S} 4$, ou seja, o consumo de oxigênio no estado basal e ativo.

A razão do controle respiratório ( $\mathrm{RCR}$ - relação entre a velocidade de consumo de oxigênio na presença de ADP e a velocidade obtida após o gasto final de ADP) e a relação ADP/O foram calculadas como índices das funções oxidativas e fosforilativas das mitocôndrias ${ }^{(2)}$.

A relação ADP/O representa a razão de ADP utilizado para fosforilação sobre o oxigênio consumido na reação, ou seja, o quanto de oxigênio foi gasto na fosforilação.

O estado $3(\mathrm{~S} 3)$ e o estado $4(\mathrm{~S} 4)$ da respiração foram expressos em ng de átomos de oxigênio por miligrama de proteína mitocondrial por minuto determinada pelo método de LOWRY et al. ${ }^{(17)}$.

\section{Estudo histológico}

As alterações histológicas foram avaliadas por um patologista, sem que o mesmo tivesse conhecimento dos grupos estudados. O material foi fixado em solução de formol a $2 \%$ e corado com hematoxilina-eosina. Foram analisados os achados referentes às alterações da microvasculatura, infiltração leucocitária portal, necrose hepatocelular e hiperplasia das células de Kupfer.

\section{Permeabilidade vascular com azul de Evans}

$\mathrm{O}$ azul de Evans é um corante que, quando injetado por via intravenosa, tem grande afinidade pela albumina. Essa característica permitiu utilizá-lo como método para avaliar permeabilidade vascular, uma vez que sua concentração no tecido pulmonar lavado é maior quanto maior for o extravasamento da albumina pelo endotélio lesado. O corante foi administrado 15 minutos antes do sacrifício e foi avaliado como descrito por JANCAR et al. ${ }^{(11)}$.

\section{Dosagem de mieloperoxidase (MPO)}

A atividade da enzima mieloperoxidase (MPO) foi utilizada como indicativo da quantidade de neutrófilos no pulmão. As amostras foram armazenadas em nitrogênio líquido. No dia do ensaio para cada $\mathrm{mg}$ de peso do tecido foram adicionados $3 \mu \mathrm{L}$ de tampão de homogeneização. Os tecidos foram homogeneizados, submetidos ao ultra-som $(40 \mathrm{~Hz})$ e centrifugados a $3000 \mathrm{~g}$ por 30 min a $4^{\circ} \mathrm{C}$. Misturou-se $10 \mu \mathrm{L}$ de cada amostra, $700 \mu \mathrm{L}$ de PBS contendo $0,25 \%$ de soro albumina bovina, $500 \mu \mathrm{L}$ de tampão 
fosfato $0,1 \mathrm{M}, 100 \mu \mathrm{L}$ de solução orto-dianisidina e $100 \mu \mathrm{L}$ de solução de azida sódica a $1 \%$. Uma alíquota de $150 \mu \mathrm{L}$ de cada amostra foi colocada em uma placa de 96 poços e a densidade óptica (DO) determinada com a utilização de filtro de $490 \mathrm{~nm}$. Os resultados foram expressos em DO a $490 \mathrm{~nm}$.

\section{ANÁLISE ESTATÍSTICA}

Os resultados foram expressos como média dos valores e desvio padrão da média (DP). Para a análise dos achados histológicos foi utilizado teste de Kruskal-Wallis. Quando houve diferença estatística foi aplicado o teste de Mann-Whitney para análise dois a dois. Para determinar a significância dos demais resultados foi utilizada análise de variância (ANOVA) para análise entre os grupos e Newman-Keuls para análise entre dois grupos. $\mathrm{O}$ valor de $P<0,05$ foi considerado significante.

\section{RESULTADOS}

As repercussões locais do fenômeno de isquemia e reperfusão foram avaliadas segundo alterações observadas no fígado. Foram analisadas as dosagens séricas de aminotransferases, a função mitocondrial e avaliação histológica.

\section{Aminotransferases}

Os níveis séricos de AST e de ALT elevaram-se significativamente no G2, submetido a lesão de isquemia e reperfusão sem droga, quando comparados aos do grupo controle $(P<0,001)$. No G3, submetido a lesão de isquemia e reperfusão com a utilização do cloridrato de tirofiban, também houve elevação de aminotransferases (Tabela 1). No entanto, estes valores foram significativamente menores que os encontrados no G2 $(P<0,05)$.

\section{Respiração mitocondrial}

Foram analisados os valores obtidos no estado basal (S4) e estado ativo (S3), e das relações RCR e ADP/O, que são indicadores das funções oxidativas e fosforilativas das mitocôndrias.

TABELA 1. Determinações séricas de aminotransferases

\begin{tabular}{llll}
\hline Grupo & \multicolumn{1}{c}{ AST } & \multicolumn{1}{c}{ ALT } \\
\hline Grupo 1 & $247,2 \pm 108$ & $88,5 \pm 28,5$ & a \\
Grupo 2 & $5406 \pm 2263$ & $5169 \pm 2690$ & b \\
Grupo 3 & $3079 \pm 1826$ & $2530 \pm 1300$ & c \\
\hline
\end{tabular}

$a, b-P<0,001$

$a, c-P<0,01$

A relação RCR apresentou redução significativa no $\mathrm{G} 2$, submetido a isquemia sem tratamento, quando comparado ao $\mathrm{G} 1$, submetido a laparotomia apenas $(P<0,001)$. No G3, tratado com cloridrato de tirofiban, houve elevação significativa do RCR, quando comparado ao G2 $(P<0,001)$ (Tabela 2$)$.

A relação $\mathrm{ADP} / \mathrm{O}$ foi significativamente menor $(P<0,001)$ no grupo submetido a isquemia e sem tratamento quando comparado ao controle. Após tratamento com cloridrato de tirofiban (G3), a relação ADP/O apresentou elevação significativa $(P<0,001)$, aproximandose dos valores encontrados no grupo controle (Tabela 3 ).
TABELA 2. Determinações da RCR

\begin{tabular}{lll}
\hline Grupo & RCR & \\
\hline Grupo 1 & $3,41 \pm 0,17$ & a \\
Grupo 2 & $1,91 \pm 0,55$ & b \\
Grupo 3 & $2,86 \pm 0,46$ & $c$ \\
\hline
\end{tabular}

$a, b-P<0,001$

$a, c-P<0,05$
$b, c-P<0,001$

TABELA 3. Determinações da relação ADP/O

\begin{tabular}{ccc}
\hline Grupo & ADP/O & \\
\hline Grupo 1 & $1,93 \pm 0,03$ & a \\
Grupo 2 & $1,45 \pm 0,27$ & b \\
Grupo 3 & $1,81 \pm 0,04$ & c \\
\hline
\end{tabular}

$a, b-P<0,001$

$a, c-N S$

$b, c-P<0,001$

\section{Estudo histológico}

O grupo tratado com tirofiban apresentou achados de necrose, infiltrado inflamatório, hemorragia e microesteatose, sendo avaliados em menor proporção $(P<0,05)$ que o grupo de isquemia não tratado (G2) (Figura 1).
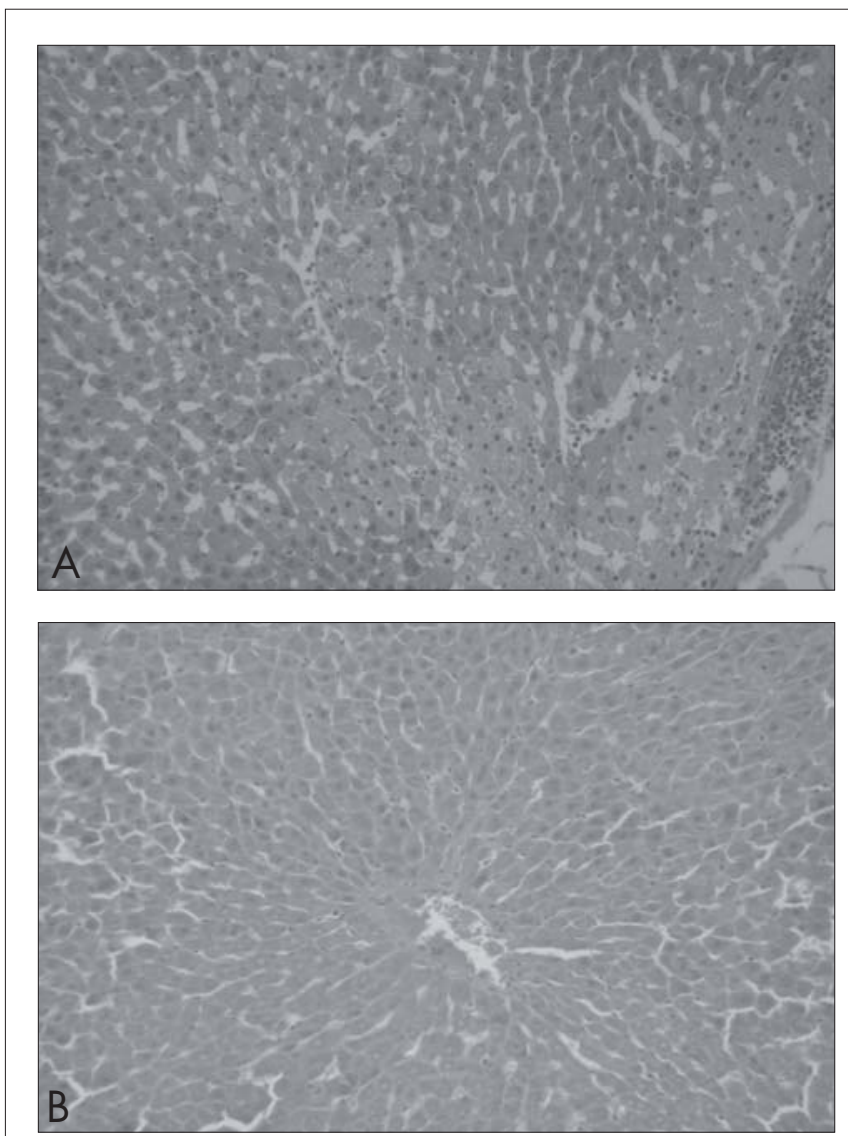

FIGURA 1. A. Estudo histológico do fígado de rato tratado com tirofiban mostra necrose centrolobular discreta. B. Aspecto microscópico de fígado de rato não tratado mostra necrose centrolobular extensa. (HE40x) 


\section{Avaliação pulmonar}

O estudo da densidade óptica do corante no parênquima pulmonar evidenciou elevação significativa nos valores obtidos no grupo submetido apenas a isquemia e reperfusão - G2 - quando comparado ao $\mathrm{G} 1$, submetido a laparotomia apenas $(P<0,01)$. No grupo tratado com cloridrato de tirofiban, a densidade óptica do corante apresentou redução significativa $(P<0,01)$, não sendo constatada diferença significativa com o G1 (Tabela 4).

\section{Mieloperoxidase}

$\mathrm{O}$ estudo da atividade da mieloperoxidase no parênquima pulmonar não demonstrou diferença significativa entre o grupo controle e o grupo submetido a isquemia e reperfusão, sem tratamento (G2). O G3 apresentou aumento significativo da atividade da mieloperoxidase no parênquima pulmonar (Tabela 5).

TABELA 4. Teste do azul de Evans

\begin{tabular}{lcl}
\hline Grupo & $\begin{array}{c}\text { Azul de Evans } \\
\text { (ug/g tecido pulmonar) }\end{array}$ & \\
\hline Grupo 1 & $194,31 \pm 53$ & $\mathrm{a}$ \\
Grupo 2 & $491,8 \pm 141$ & $\mathrm{~b}$ \\
Grupo 3 & $193,6 \pm 103,4$ & $\mathrm{c}$ \\
\hline
\end{tabular}

$a, b-P<0,01$

$a, \mathrm{c}-\mathrm{NS}$

$b, c-P<0,01$

TABELA 5. Atividade da mieloperoxidase no parênquima pulmonar

\begin{tabular}{lcl}
\hline Grupo & $\begin{array}{c}\text { Mieloperoxidase } \\
\text { (DO a 490 nm) }\end{array}$ & \\
\hline Grupo 1 & $0,029 \pm 0,005$ & a \\
Grupo 2 & $0,053 \pm 0,027$ & b \\
Grupo 3 & $0,107 \pm 0,041$ & $\mathrm{c}$ \\
\hline
\end{tabular}

$\mathrm{a}, \mathrm{b}-\mathrm{NS}$

$a, c-P<0,00$
$b, c-P<0,01$

\section{DISCUSSÃO}

A lesão de isquemia e reperfusão hepática é comumente encontrada em uma variedade de condições clínicas como o transplante hepático, hepatectomias ${ }^{(3,18,19,24)}$ e estados póschoque. A importância dada a esse fenômeno é cada vez maior, especialmente no transplante hepático. Atualmente, a lesão de isquemia e reperfusão é a principal variável responsável pelos resultados precoces do transplante hepático.

A participação das plaquetas na lesão de isquemia e reperfusão tem papel destacado ${ }^{(14)}$. Esta participação na fisiopatogenia da lesão de isquemia e reperfusão do coração ${ }^{(8)}$, pulmão $^{(20)}$ e pâncreas ${ }^{(15)}$ está bem estabelecida. No fígado, CYWES et al. ${ }^{(5,}$ 6) demonstraram, em trabalhos clínico e experimental, que a lesão de reperfusão hepática se correlaciona diretamente com a ativação das plaquetas.

Os efeitos do cloridrato de tirofiban na lesão de isquemia e reperfusão hepática foram avaliados utilizando-se os níveis séricos das aminotransferases, a análise da função mitocondrial e exame histológico do fígado. O teste de azul de Evans e dosagem tecidual de mieloperoxidase foram empregados para o estudo dos efeitos pulmonares.

Encontrou-se lesão hepatocelular importante no grupo G2, submetido a isquemia e reperfusão sem tratamento, que apresentou níveis séricos de aminotransferases significativamente superiores ao grupo G1. Com o uso do cloridrato de tirofiban, o G3 apresentou níveis de aminotransferases significativamente inferiores ao $\mathrm{G} 2$, o que denota efeito protetor da droga na lesão de isquemia e reperfusão.

A avaliação da função mitocondrial é estudo mais sensível que os exames laboratoriais. A mitocôndria é a principal organela da respiração celular e oferece o substrato energético das células, a adenosina trifosfato (ATP). Portanto, o funcionamento adequado dessa organela é fundamental para a viabilidade do tecido e por este motivo é utilizado em diversos modelos experimentais.

Em recente revisão, JASSEM et al. ${ }^{(12)}$ discutiram o papel da mitocôndria na lesão de isquemia e reperfusão. Esses autores ressaltaram a importância da mitocôndria no estresse oxidativo, grande responsável pela lesão tecidual aguda ${ }^{(1,10,25)}$.

O presente estudo mostrou redução significativa dos valores do estado ativo (S3) no grupo de ratos submetidos a isquemia e reperfusão quando comparados ao grupo controle. Essa diminuição reflete a redução da capacidade de utilização do oxigênio para produzir ATP a partir do ADP. No grupo tratado com cloridrato de tirofiban, os valores de S3 se elevaram, demonstrando efeito protetor desta droga sobre a função de fosforilação mitocondrial. No estado basal (S4), por sua vez, não houve variações significativas nos três grupos estudados. $\mathrm{O}$ aumento do consumo de oxigênio em $\mathrm{S} 4$ e a ausência de resposta ao estímulo com ADP, estão associados ao desacoplamento da oxidação e fosforilação mitocondrial. Uma vez desacoplada, a mitocôndria consome oxigênio de forma independente em níveis basais superiores aos normais. O desacoplamento é processo irreversível e letal. Como não houve diferenças significativas entre os três grupos, conclui-se que o modelo utilizado produz lesão celular significativa, contudo, reversível.

A lesão de isquemia e reperfusão leva a uma série de alterações relacionadas à falta de aporte de nutrientes para as células, seguida de resposta inflamatória desencadeada pelo restabelecimento da circulação ${ }^{(10)}$. Na microcirculação, uma série de eventos leva à agregação plaquetária, migração dos leucócitos para o interstício, liberação de radicais livres de oxigênio e lesão tecidual. Essa agressão pode levar a lesões irreversíveis do tecido ou a alterações ultra-estruturais que modificam o funcionamento normal da célula, sem, necessariamente, causar danos letais. Assim, a perda do metabolismo normal dos hepatócitos leva a acúmulo intracelular de lípides e a perda da função da bomba de sódio-ATP possibilita a passagem de água para o meio intracelular, levando à tumefação dos hepatócitos ${ }^{(12)}$. Esses hepatócitos vacuolizados e tumefeitos favorecem o aumento da resistência portal intrahepática, isquemia e, conseqüentemente, lesão tecidual ${ }^{(1)}$. O grupo de ratos submetidos a isquemia e reperfusão (G2) apresentou redução significativa da capacidade de fosforilação das mitocôndrias dos hepatócitos, quando comparado ao grupo controle (G1). O cloridrato de tirofiban demonstrou melhora significante no consumo da ADP, formando mais moléculas 
do substrato energético celular, ATP. Dessa forma, o estudo da função da ultra-estrutura celular responsável pelo fornecimento energético indispensável à viabilidade dos hepatócitos mostrou que o antagonista da glicoproteína IIb/IIIa teve efeito protetor neste modelo experimental.

Os resultados do presente estudo demonstraram aumento significativo na intensidade de necrose, infiltrado de células inflamatórias, microesteatose e hemorragia sinusoidal no grupo de ratos submetido a isquemia e reperfusão. No grupo tratado com cloridrato de tirofiban, essas variáveis apresentaram diminuição significativa da intensidade, o que, mais uma vez, demonstra efeito protetor para a lesão de isquemia e reperfusão.

As repercussões da lesão de isquemia e reperfusão hepática não se restringem ao fígado, uma vez que o estado próinflamatório resultante da reperfusão é capaz de alterar outros órgãos não submetidos a lesão isquêmica. Um dos órgãos mais afetados é o pulmão, que por sua vez, tem grande impacto na sobrevida do doente ${ }^{(20)}$. O teste com azul de Evans é indicador da permeabilidade vascular. A alta afinidade do contraste com a albumina possibilita quantificar a lesão endotelial pela densidade óptica do tecido. Os resultados obtidos no presente trabalho, demonstraram alteração significativa da permeabilidade vascular dos pulmões dos ratos submetidos a isquemia e reperfusão do fígado. O uso do antagonista da glicoproteína IIb-IIIa, cloridrato de tirofiban, evitou a lesão endotelial da microcirculação pulmonar, demonstrando o seu efeito protetor na lesão de reperfusão em órgãos à distância. Esses dados também sugerem que as plaquetas têm um papel importante na fisiopatogenia da lesão de reperfusão. Contudo, como demonstrado nos resultados da atividade da mieloperoxidase no tecido pulmonar do grupo tratado com cloridrato de tirofiban, o aumento significativo da sua atividade comprova que existe uma relação intercelular importante entre as plaquetas e os leucócitos, e que estes não são os únicos responsáveis pela lesão pulmonar de reperfusão.

Concluiu-se que o uso do cloridrato de tirofiban exerceu papel protetor da lesão hepática de isquemia e reperfusão, com melhora dos parâmetros histológicos, bioquímicos e da respiração mitocondrial dos hepatócitos e impede o aumento da permeabilidade vascular secundária à lesão de reperfusão hepática.

\section{AGRADECIMENTOS}

Os autores agradecem a participação efetiva das Sras. Nilza A. T. Molan e Ana Lúcia Bernardes nas dosagens bioquímicas deste trabalho.

Canedo LF, Machado MAC, Coelho AMM, Sampietre SN, Bacchella T, Machado MCC. Protective effects of an inhibitor of glycoprotein IIb/IIIa in the hepatic and pulmonary disturbances secondary to ischemia and reperfusion injury of rat's liver. 2007;44(3):276-81.

ABSTRACT - Background - Hepatic ischemia-reperfusion injury is responsible for a considerable morbidity and mortality. Aim - To evaluate the effect of a platelet glycoprotein IIb/IIIa receptor inhibitor (tirofiban) on hepatic and pulmonary disturbances associated with hepatic ischemia-reperfusion injury. Methods - Twenty-three Wistar rats divided in three groups: rats sham-operated $(\mathrm{n}=6)$, rats submitted to ischemia-reperfusion that received saline solution $(\mathrm{n}=8)$, and rats submitted to ischemia-reperfusion treated with $0.7 \mathrm{mg} / \mathrm{kg}$ of tirofiban $(\mathrm{n}=9)$. Serum aminotransferases $($ AST and ALT) were also determined, and the study of hepatic tissue histology was carried out. The evaluation of the pulmonary disturbances was done using the Evans blue test and the tissular determination of myeloperoxidase. Hepatic mitochondrial oxidation and phosphorylation were also measured. Results - There was an increase in the state 3 respiration, $\mathrm{ADP} / \mathrm{O}$ ratio and respiration control rate in the group treated with tirofiban. This group had also lower levels of aminotransferases and the histological findings were significantly less intense. Pulmonary evaluation demonstrated decrease of the Evans blue test in the tirofiban group and an increase of its tissular determination of myeloperoxidase. Conclusions - The inhibition of glycoprotein IIb/IIIa receptor with tirofiban protected the hepatic disturbances and prevented the increase of pulmonary vascular permeability secondary to the ischemia-reperfusion injury of the liver.

HEADINGS - Reperfusion injury. Liver. Hepatectomy. Platelet membrane glycoproteins. Rats. 


\section{REFERÊNCIAS}

1. Caraceni P, Ryu HS, Van Thiel DH, Borle AB. Source of oxygen free radicals produced by rat hepatocytes during postanoxic reoxygenation. Biochim Biophys Acta. $1995 ; 21: 249-54$.

2. Chance B, Williams GR. A simple and rapid assay of oxidative phosphorylation. Nature. 1955;175: 1120-1.

3. Clavien PA, Harvey PR, Strasberg SM. Preservation and reperfusion injuries in liver allografts. An overview and synthesis of current studies. Transplantation. 1992;53: 957-78.

4. Coelho AM, Machado MC, Sampietre SN, Leite KR, Molan NA, Pinotti HW. Hepatic lesion in experimental acute pancreatitis Influence of pancreatic enzyme storage reduction. Rev Hosp Clin Fac Med São Paulo. 1998;53:104-9.

5. Cywes R, Mullen JB, Stratis MA, Greig PD, Levy GA, Harvey PR, Strasberg SM. Prediction of the outcome of transplantation in man by platelet adherence in donor liver allografts: evidence of the importance of prepreservation injury. Transplantation 1993;56:316-23.

6. Cywes R, Packham MA, Tietze L, Sanabria JR, Harvey PR, Phillips MJ, Strasberg SM. Role of platelets in hepatic allograft preservation injury in the rat. Hepatology 1993;18:635-47.

7. Estabrook K. Mitochondrial respiratory control and polarographic measurement ADP/O ratios. Methods Enzymol. 1964:41-7.

8. Flores NA, Goulielmos NV, Seghatchian MJ, Sheridan DJ. Myocardial ischaemia induces platelet activation with adverse electrophysiological and arrhythmogenic effects. Cardiovasc Res. 1994;28:1662-71.

9. Furukawa H, Todo S, Imventarza O, Casavilla A, Wu YM, Scotti-Foglieni C, Broznick B, Bryant J, Day R, Starzl TE. Effect of cold ischemia time on the early outcome of human hepatic allografts preserved with UW solution. Transplantation 1991;1:1000-4

10. Gonzalez-Flecha B, Cutrin JC, Boveris A. Time course and mechanism of oxidative stress and tissue damage in rat liver subjected to in vivo ischemia-reperfusion. J Clin Invest. 1993;91:456-64.

11. Jancar S, De Giaccobi G, Mariano M, Mencia-Huerta JM, Sirois P, Braquet P. Immune complex induced pancreatitis: effect of BN 52021 a selective antagonist of plateletactivating factor. Prostaglandins. 1988;35:757-70.

12. Jassem W, Fuggle SV, Rela M, Koo DD, Heaton ND. The role of mitochondria in ischemia/reperfusion injury. Transplantation. 2002;27:493-9.
13. Johnson PC, Sheppeck RA, Hribar SR, Bentz ML, Janosky J, Dickson CS. Inhibition of platelet retention on artificial microvascular grafts with monoclonal antibodies and a high-affinity peptide directed against platelet membrane glycoproteins. Arterioscler Thromb. 1991;11:552-60.

14. Khandoga A, Biberthaler P, Enders G, Axmann S, Hutter J, Messmer K, Krombach F. Platelet adhesion mediated by fibrinogen-intercelllular adhesion molecule-1 binding induces tissue injury in the postischemic liver in vivo. Transplantation. 2002;74:681-8.

15. Kuroda T, Shiohara E, Homma T, Furukawa Y, Chiba S. Effects of leukocyte and platelet depletion on ischemia-reperfusion injury to dog pancreas. Gastroenterology. 1994; 107:1125-34.

16. Lefer AM, Weyrich AS, Buerkem Role of selectins a new family of adhesion molecules in ischemia-reperfusion injury. Cardiovasc Res. 1994;28:289-94.

17. Lowry OH, Rosebrough NJ, Farr AL, Randall RJ. Protein measurement with the Folin phenol reagent. J Biol Chem. 1951;193:265-75.

18. Machado MA, Herman P, Machado MC. A standardized technique for right segmental liver resections. Arch Surg. 2003;138:918-20.

19. Machado MA, Herman P, Machado MC. Anatomical resection of left liver segments. Arch Surg. 2004;139:1346-9.

20. Okada Y, Marchevsky AM, Zuo XJ, Pass JA, Kass RM, Matloff JM, Jordan SC Accumulation of platelets in rat syngeneic lung transplants: a potential factor responsible for preservation-reperfusion injury. Transplantation. 1997;64:801-6.

21. Peerschke EI, Zucker MB, Grant RA, Egan JJ, Johnson MM. Correlation between fibrinogen binding to human platelets and platelet aggregability. Blood. 1980;55:841-7.

22. Phillips DR, Charo IF, Parise LV, Fitzgerald LA. The platelet membrane glycoprotein IIb-IIIa complex. Blood. 1988;71:831-43.

23. Ploeg RJ, D'alessandro AM, Knechtle SJ, Stegall MD, Pirsch JD, Hoffmann RM, Sasak T, Sollinger HW, Belzer FO, Kalayoglu M. Risk factors for primary dysfunction after liver transplantation--a multivariate analysis. Transplantation. 1993;55:807-13.

24. Pringle JH. Notes on the arrest of hepatic hemorrhage due to trauma. Ann Surg. 1908; $48:$ : 541-9

25. Schild L, Reinheckel T, Wiswedel I, Augustin W. Short-term impairment of energy production in isolated rat liver mitochondria by hypoxia/reoxygenation: involvement of oxidative protein modification. Biochem J. 1997;328:205-10.

Recebido em 22/9/2006. Aprovado em 10/5/2007. 